Revue internationale P.M.E.

Économie et gestion de la petite et moyenne entreprise

\title{
Prestations de conseil auprès des PME/PMI et processus d'apprentissage de leurs dirigeants : pour une autre conception du métier de conseil en management
}

\section{Daniel Belet}

Volume 6, numéro 2, 1993

URI : https://id.erudit.org/iderudit/1008212ar

DOI : https://doi.org/10.7202/1008212ar

Aller au sommaire du numéro

Éditeur(s)

Presses de l’Université du Québec

ISSN

0776-5436 (imprimé)

1918-9699 (numérique)

Découvrir la revue

Citer cette note

Belet, D. (1993). Prestations de conseil auprès des PME/PMI et processus d'apprentissage de leurs dirigeants : pour une autre conception du métier de conseil en management. Revue internationale P.M.E., 6(2), 109-134. https://doi.org/10.7202/1008212ar
Résumé de l'article

Partant du constat du développement du marché du conseil d'entreprise et du flou régnant autour de ce nouveau métier; l'auteur s'interroge sur son essence et sur les pratiques professionnelles qui le caractérisent. Prenant appui sur sa propre expérience de consultant, il souligne les nombreuses ambiguïtés qui apparaissent dans l'exercice courant de ce métier, en particulier, pour les interventions auprès des dirigeants de PME/PMI qui sont en général moins formalisées que dans les plus grandes organisations.

Il montre que ces difficultés sont dues, d'une part, à l'absence fréquente de maîtrise par l'intervenant des dimensions relationnelles et psychologiques spécifiques au dirigeant de PME/PMI et, d'autre partf à une approche de ce métier de conseil en termes de contenu ou d'expertise plutôt qu'en termes de processus de développement.

L'auteur propose une approche "pédagogique » du conseil d'entreprise à partir de l'exemple de la clientèle des dirigeants de PME/PMI. L'intervention du conseil est alors considérée essentiellement comme une action de développement des processus d'apprentissage du client. Elle a le mérite de situer les prestations de conseil dans un nouveau cadre conceptuel permettant non seulement de clarifier les relations entre le consultant et son client, mais aussi de préciser et justifier une autre " valeur ajoutée » du consultant.

Cette perspective implique la maîtrise de nouvelles compétences professionnelles par les consultants qui ne font pas actuellement l'objet ni de formations ni la plupart du temps de pratiques adéquates. Elle conduit aussi à un autre regard sur la profession de conseil d'entreprise en milieu PME/PMI et à ses besoins de progrès qualitatifs à l'avenir.
Ce document est protégé par la loi sur le droit d'auteur. L'utilisation des services d’Érudit (y compris la reproduction) est assujettie à sa politique d'utilisation que vous pouvez consulter en ligne.

https://apropos.erudit.org/fr/usagers/politique-dutilisation/ 
Note de recherche

\title{
Prestations de conseil auprès des PME/PMI et processus d'apprentissage de leurs dirigeants: pour une autre conception du métier de conseil en management
}

Daniel BELET *

Université de Bordeaux

\begin{abstract}
RÉSUMÉ
Partant du constat du développement du marché du conseil d'entreprise et du flou régnant autour de ce nouveau métier, l'auteur s'interroge sur son essence et sur les pratiques professionnelles qui le caractérisent. Prenant appui sur sa propre expérience de consultant, il souligne les nombreuses ambiguittés qui apparaissent dans l'exercice courant de ce métier, en particulier, pour les interventions auprès des dirigeants de PME/PMI qui sont en général moins formalisées que dans les plus grandes organisations.

II montre que ces difficultés sont dues, d'une part, à l'absence fréquente de maîtrise par l'intervenant des dimensions relationnelles et psychologiques spécifiques au dirigeant de PME/PMI et, d'autre part, à une approche de ce métier de conseil en termes de contenu ou d'expertise plutôt qu'en termes de processus de développement.
\end{abstract}

* Daniel Belet détient un doctorat ès sciences économiques, un doctorat de $3^{\mathrm{e}}$ cycle en gestion et un M.S. en management de l'Université Northwestern (USA). Il est consultant en management et professeur-associé à l'Université de Bordeaux. Il est cofondateur et président de l'ADEFMA (Association pour le développement de la formation au management), organisme spécialisé en ingénierie pédagogique du management. Adresse : B.P. 16, chemin de Guillaumot, 33650 La Brede, France.

L'auteur remercie Inès de la Ville pour sa contribution sur les thèmes des apprentissages individuel et organisationnel sur lesquels elle travaille dans le cadre de sa thèse de doctorat en sciences de gestion. 
L'auteur propose une approche "pédagogique " du conseil d'entreprise à partir de l'exemple de la clientèle des dirigeants de PME/PMI. L'intervention du conseil est alors considérée essentiellement comme une action de développement des processus d'apprentissage du client. Elle a le mérite de situer les prestations de conseil dans un nouveau cadre conceptuel permettant non seulement de clarifier les relations entre le consultant et son client, mais aussi de préciser et justifier une autre "valeur ajoutée " du consultant.

Cette perspective implique la maîtrise de nouvelles compétences professionnelles par les consultants qui ne font pas actuellement l'objet ni de formations ni la plupart du temps de pratiques adéquates. Elle conduit aussi à un autre regard sur la profession de conseil d'entreprise en milieu PME/PMI et à ses besoins de progrès qualitatifs à l'avenir.

\section{ABSTRACT}

Noticing the management consulting growth but also the fuzziness that characterize this rather new profession in France, the author is wondering about its very essence and about its current practices. Leaning upon his own consulting experience, he stresses the numerous ambiguities that often appear in the practice of this job, especially with small business executives, with whom the relationship are usually more informal and slack than with large organizations.

He shows that these difficulties are very often the consequences of the consultant inadequate mastership of the necessary psychological and communication skills to deal with small business executives. They are also due to a resources or expertise oriented consulting approach rather than a learning process oriented consulting approach.

The author proposes a "pedagogical" approach of management consulting with small business executives. The management consulting job is then focused on the management learning process of the client. This perspective has the advantage to give to the management consulting profession an other main conceptual framework allowing not only a clarification of the relationship between the consultant and his small business client but also a new legitimacy for the "added value" of the consulting services.

But this learning approach does suppose the mastership of new specific professional competences by the management consultants for which they are not yet well trained, at least within the french environment.

It also leads to another look at the profession of small business management consulting and it emphasizes its qualitative improvement needs in the future.

\section{RESUMEN}

Constatando el reciente desarrollo del mercado de los consultores de empresa y de la imprecision que reina entorno a este nuevo oficio, el autor se interrroga a cerca de su verdadera definicion $y$ de sus practicas profesionales 
caracteristicas. Apoyandose sobre su propia experiencia como consultor, subraya las multiples ambiguedades que aparecen en la practica de este oficio. Especialmente, en sus aplicaciones con los dirigentes de PME/PMI, que por lo general son menos estructuradas que en organizaciones mayores.

Señala que estas dificultades se deben, por una parte, a la frecuente ausencia de dominio por parte del consultor de las dimensiones relacionales y psicologicas especificas del dirigente de PME/PMI, y por otra parte, a un enfoque de este oficio orientado hacia el contenido o la pericia técnica mas bien que hacia los processos de desarrollo.

El autor propone un enfoque pedagogico de la consultoria de empresas, fundandose sobre el ejemplo de los dirigentes de PME/PMI. La intervencion del consultor se presenta entonces esencialmente como una accion destinada a fomentar los procesos de aprendizaje del cliente. Tiene el merito de situar las prestaciones de consultoria de empresas en un nuevo marco conceptual que permite a la vez clarificar las relaciones entre el consultor y su cliente, asi mismo, justificar otro " valor añadido " del consultor.

Este enfoque supone para los consultores dominar nuevas competencias profesionales a las que de momento, no corresponde ninguna formacion, ni en la mayoria de los casos, practica adecuada. Lo que significa concebir de otra forma la profesion de consultor en pequeñas y medianas empresas y tambien sus necesidades de desarrollo calitativo en el futuro.

\section{Introduction}

Depuis une quinzaine d'années, l'activité de conseil d'entreprise aux PME/PMI s'est considérablement développée en France notamment grâce aux procédures d'aides financières mises en place par les pouvoirs publics (FRAC, ANVAR, etc.) pour consolider un tissu économique créateur d'emplois ${ }^{1}$. La croissance de ce marché du conseil aux PME/PMI a suscité l'émergence, voire la prolifération d'une offre de cabinets-conseils d'entreprises. À ces nouveaux venus s'est d'ailleurs ajoutée l'offre de certains cabinets-conseils plus anciens qui se sont intéressés à ce nouveau segment du marché du conseil.

Comme toute profession relativement nouvelle et ouverte, les prestations de conseil aux PME/PMI ont été pratiquées par des «consultants » d'origines professionnelles, de formation, d'expérience et de compétences très diverses et très hétérogènes. Le métier de conseil d'entreprise (notamment auprès des

1. Voir à ce sujet les enquêtes réalisées par le cabinet Bernard-Julhiet Conseils (1991), Engel et Fixari (1987), Atamer, Durand, Riot, Engel et Fixari (1989), Sauviat (1989). 
PME/PMI) ne faisant pas l'objet d'une formation spécifique reconnue ${ }^{2}$ est souvent exercé de façon très différente par les praticiens.

Il convient de bien souligner la spécificité de l'activité de conseil aux PME/PMI par rapport aux grandes entreprises dans le contexte français à cause des caractéristiques psychologiques et culturelles particulières des dirigeants de PME/PMI : prédominance des relations directes avec le dirigeant et secondairement ses proches collaborateurs, modes d'appréhension concrets et à court terme des problèmes de l'entreprise, poids de la personnalité et du style de management personnel du dirigeant, habitude du dirigeant de se débrouiller seul, méfiance fréquente et scepticisme vis-à-vis d'intervenants extérieurs, etc. Ces particularités font que le conseil en management auprès de PME/PMI est un métier délicat, nécessitant un minimum de compétences de "généraliste» et souvent difficile à exercer de façon satisfaisante et efficace. Il constitue en réalité une véritable spécialité professionnelle parmi les activités de conseil d'entreprise'.

La source principale des difficultés de cette profession ne réside pas dans les aspects techniques de la relation de conseil, en général relativement simples au niveau des PME/PMI (et qui donnent d'ailleurs l'impression d'un accès aisé et d'une pratique relativement facile de ce métier). Elle provient essentiellement des aspects relationnels et psychologiques de la prestation du conseil vis-à-vis de son client dirigeant de PME/PMI, et de la façon dont il gère cette relation dans le cadre de son intervention.

Ces dimensions relationnelles sont souvent la cause d'attentes déçues, de malentendus, conduisant à des interventions sans résultats probants aux yeux du client et frustrantes pour l'intervenant. Mais elles expliquent aussi le relatif succès pour le dirigeant de PME/PMI de missions de conseil (au-delà des aspects techniques et de la rationalisation a posteriori de l'action du conseil qu'il fera) liées à des réponses, à des besoins psychologiques implicites (tels que l'écoute, la validation d'une analyse, la formation à une technique managériale, l'échange sur des problèmes le préoccupant, etc.).

2. Voir ISEOR (Institut de socio-économie des entreprises et des organisations) de l'Université de Lyon 2 dirigé par H. Savall ; IDCE à Angers de création récente (niveau bac +3 et orienté vers les PME) ; un DESS à l'Université de ParisDauphine (surtout orienté vers la formation), une maîtrise à l'ESC Nantes-Atlantique ouvert à la rentrée 92 , mais elles ne touchent encore que très marginalement la profession du conseil d'entreprise. Rien n'existe en revanche actuellement, à notre connaissance, dans le domaine des formations continues longues au métier de conseil en management.

3. L'auteur fonde cette observation sur son expérience de conseil d'une douzaine d'années auprès de nombreuses PME/PMI. 
Dans un souci d'amélioration de la qualité du conseil aux PME/PMI, il paraît nécessaire de mieux appréhender et de traiter de façon plus «professionnelle » cette dimension relationnelle et psychologique du métier de conseil d'entreprise. Par ailleurs, compte tenu de la relative jeunesse de ce métier, il paraît utile de proposer de nouvelles approches théoriques susceptibles de contribuer à le «professionnaliser» davantage et à améliorer son image et son efficacité aux yeux des dirigeants d'entreprise.

Une approche paraissant particulièrement intéressante pour étudier la relation entre le conseil et les dirigeants de PME/PMI a trait à ses dimensions «pédagogiques». Dans cette perspective, la relation de conseil peut être considérée sous l'angle de la maîtrise des processus d'apprentissage du client à l'aide de nouveaux concepts, de savoir-faire psycho-pédagogique, d'outils méthodologiques, etc. qui permettent d'agir sur ses perceptions, ses représentations et ses schémas mentaux, et donc sur ses attitudes, ses comportements, ses raisonnements et ses principes d'action ${ }^{4}$.

Nous nous attacherons spécialement, dans le cadre restreint de cet article, à ces aspects «pédagogiques» de la relation de conseil qui constituent à nos yeux, un élément clé de sa réussite en observant qu'ils sont généralement occultés dans les prestations de conseil (et même souvent de formation) aux dirigeants de PME/PMI ${ }^{5}$. Ceux-ci nous paraissent particulièrement déterminants dans le cas de la relation entre le dirigeant de PME/PMI et le consultant, car cette relation est toujours très personnalisée et chargée d'un contenu émotionnel important (ce qui est en général beaucoup moins le cas dans une grande organisation). Ce dernier pèsera sur la perception de cette relation par le client et conditionnera largement les effets qui en résulteront.

4. En réalité, il existe au moins 2 types de processus d'apprentissage dans une relation de conseil :

- des processus de nature individuelle: celui du dirigeant (et de ses collaborateurs le cas échéant), et celui du conseil ;

- des processus de nature collective : celui de l'entreprise ou de groupes de personnes dans l'organisation.

Ceci dit, nous n'aborderons ici, dans un souci de simplification et compte tenu du thème majeur de cet article, que le seul type de processus d'apprentissage concernant le dirigeant d'entreprise dans le cadre de sa relation avec le conseil.

5. Observons que la distinction traditionnelle entre activité de «conseil» et activité de «formation » apparaît comme artificielle et éloignée de la réalité, surtout dans le contexte des PME/PMI. Il existe toujours dans une relation de conseil réussie une certaine dimension de formation. Conseil et formation sont donc en pratique intimement liés et contribuent en fait tous deux aux processus d'apprentissage de l'entreprise et de son dirigeant. 
Précisons bien qu'il n'est pas naturellement question de critiquer ou de sous-estimer le rôle d'apport d'expertise du conseil, surtout du conseil spécialisé dans une discipline donnée et intervenant pour un problème ponctuel à caractère technique. Mais nous avons choisi de nous intéresser ici essentiellement à la fonction du conseil en management «généraliste » auprès des PME/ PMI et donc essentiellement de leurs dirigeants.

Les relations entre le conseil en management et les dirigeants de PME/PMI conduisent souvent à diverses ambiguittés d'origine relationnelle et psychologique qui nuisent à l'efficacité de la prestation de conseil. C'est pourquoi nous proposons une approche «pédagogique» de la relation de conseil au dirigeant de PME/PMI axée sur le développement d'un processus d'apprentissage. Son intérêt est de permettre une clarification de la relation de conseil et d'éviter certains obstacles psychologiques qu'elle suscite. De plus, elle a le mérite d'offrir une possibilité de renouvellement de la philosophie de ce métier encore jeune. On proposera dans cette perspective une autre conception du métier de consultant auprès des PME/PMI qui conduit à s'interroger sur la nature des compétences nécessaires à son exercice notamment aux plans psycho-pédagogique et de la communication. Le développement de ces compétences semble être la clé de la professionnalisation de ce métier de conseil en management auprès des PME/PMI et une voie d'amélioration de la qualité des prestations de conseil dans les années à venir.

\section{Les ambiguiités de la relation entre le dirigeant de PME/PMI et le conseil}

Le métier de conseil d'entreprise, en l'absence de codification et de normalisation professionnelles précises, se caractérise en France par une nébuleuse rassemblant toutes sortes d'intervenants et de pratiques professionnelles (allant des plus fantaisistes... aux plus sérieuses!). Il souffre indéniablement d'un ensemble d'ambiguïtés qu'il paraît intéressant de souligner si l'on veut être en mesure de proposer des clarifications salutaires, d'améliorer la qualité et l'efficacité des prestations et, par conséquent, de contribuer à «professionnaliser» ce métier de conseil.

\subsection{Ambiguittés dans les motivations du recours au conseil}

De nombreuses enquêtes ${ }^{6}$ effectuées sur le thème du conseil au PME/PMI en France montrent que:

6. Notamment l'enquête la plus récente du cabinet Bernard-Julhiet Conseils. 
- la demande des chefs d'entreprise est motivée par des raisons très diverses (manque de compétences internes, souhait d'un œil neuf, manque de temps, recherche d'une aide financière, etc.);

- les circonstances sont aussi très variées, mais souvent floues et générales (modifications de l'environnement, pression de la clientèle, réorganisation, investissement, difficultés, etc.);

- en réalité, dans de nombreux cas, les PME/PMI ont recours au conseil malgré eux sous la pression d'un prescripteur (DRIRE, ANVAR, CCI, banques, etc.) ou la recommandation d'un collègue.

On constate donc très fréquemment chez ce type de clientèle des motivations initiales plutôt ambiguës et floues pour le recours aux services d'un conseil. À cela s'ajoutent certaines craintes vis-à-vis d'un conseil extérieur légitimées par l'hétérogénéité des intervenants ${ }^{7}$, le manque de lisibilité et les difficultés d'appréciation de leurs réelles compétences et références.

La recherche d'une relation de confiance (Atamer et al., 1989) avec le conseil l'emporte souvent sur la recherche d'une réelle compétence et expérience professionnelle délicate à évaluer pour le dirigeant de PME/PMI; et cela contribue à l'ambiguité de la relation avec celui-ci.

Enfin, on observe qu'il existe très rarement des cahiers de charges précis fixés par les dirigeants de PME/PMI, ce qui crée une source d'ambiguïté supplémentaire, même si cela peut se justifier par un besoin de flexibilité de l'intervention au fur et à mesure de son déroulement dans le temps, souvent d'ailleurs en fonction des problèmes prioritaires perçus par le dirigeant.

\subsection{Ambiguités dans les attentes du dirigeant de PME/PMI par rapport au conseil}

On observe que les dirigeants de PME/PMI ont des attentes implicites variées et parfois précises vis-à-vis d'un conseil : souvent l'apport d'un avis d'expert et d'une solution technique à un problème précis, une caution pour avaliser des décisions déjà prises, la validation de certaines options ou orientations envisagées et plus rarement le point de vue objectif, car extérieur, d'un regard de généraliste compétent et expérimenté sur leur entreprise.

7. En dépit des efforts faits par certains organismes (DRIRE, CCI, ANVAR, FRAC, etc.) pour prescrire des listes de conseils d'entreprises fiables et compétents. Remarquons que les critères d'évaluation des cabinets par ces organismes restent discutables et n'empêchent pas, dans la réalité, des recommandations parfois plus « amicales » que fondées sur de réelles compétences professionnelles ou des références sérieuses. 
Les dirigeants de PME/PMI sollicitant un conseil ont souvent tendance à attendre une obligation de résultats, alors qu'en règle générale, un conseil d'entreprise se situe dans une logique d'obligation de moyens seulement; ce qui explique aussi de fréquents malentendus et pose le problème délicat des limites de son intervention, de ses responsabilités et de la façon dont il doit gérer son intervention (Stevenson et Sahlman, 1988; Noye et Verne, 1991).

\subsection{Ambiguiltés dans l'attitude du dirigeant de PME/PMI vis-à-vis du conseil d'entreprise}

Dans le contexte français des PME/PMI, le dirigeant, souvent créateur de l'entreprise, sait par définition tout faire et se débrouille comme il peut, sans faire appel à l'extérieur - dans la mesure du possible.

Ce phénomène est renforcé par une tradition d'individualisme et de secret des petites entreprises françaises méfiantes en général, par principe, vis-à-vis toute ingérence extérieure.

Il est encore mal admis parmi les dirigeants de PME/PMI (bien que cela change progressivement, notamment sous l'impact des procédures d'aides financières publiques telles que les FRAC) d'avoir recours spontanément à un conseil extérieur lorsqu'on est confronté à un problème qu'on ne peut pas résoudre soi-même ou au moyen des compétences internes de façon optimum ${ }^{8}$.

Il y a donc pour de nombreux dirigeants de PME/PMI un certain aspect «humiliant » à avoir recours à un conseil extérieur et, par conséquent, une barrière psychologique à franchir pour l'intervenant à ce niveau-là.

\subsection{Ambiguïté sur le «positionnement " professionnel du conseil}

Schématiquement, on peut distinguer plusieurs catégories de conseils intervenant auprès des PME/PMI. Le critère intéressant n'est pas leur statut juridique (indépendant, membre d'un cabinet-conseil, d'un organisme parapublic, d'une institution de formation, etc.), mais les conceptions dominantes de leurs pratiques professionnelles. Dans cet esprit, on peut distinguer les principaux profils suivants :

- l'expert technicien (conseil juridique, expert-comptable, conseil marketing, conseil en gestion, conseil en recrutement, etc.) spécialiste d'une discipline ou fonction de l'entreprise ;

8. Selon l'enquête de 1991 du cabinet Bernard-Julhiet Conseils (1991), $54 \%$ des PME/PMI françaises déclaraient n'avoir jamais eu recours à un conseil extérieur ! 
- le spécialiste sectoriel : industrie du bois, des plastiques, de la mécanique, de l'électronique, etc. (en général avec une expérience du secteur de plusieurs années);

- le conseil généraliste PME/PMI (avec en général une expérience de direction générale);

- le formateur-conseil (dont l'approche est basée sur le plan de formation de l'entreprise);

- le conseil-«gourou» (souvent adepte de théories de développement personnel et recherchant une influence psychologique directe sur le dirigeant d'entreprise).

En pratique, les styles de conseil sont rarement clairs et comprennent un mélange des genres (Kubr, 1981; Khan, 1990 ; Bounfour, 1989), source d'ambiguiités et parfois de frustrations tant pour les clients que pour les conseils eux-mêmes. Ces derniers ont en effet souvent du mal à se positionner clairement par rapport à la demande explicite et surtout implicite de leurs clients qui comprend fréquemment un non-dit important d'attentes psychologiques.

Enfin, il convient d'observer que les compétences réelles des conseils et leurs domaines de spécialité sont souvent mal perçus par les clients, reflétant ainsi un indéniable problème de communication de cette profession.

\subsection{Ambiguïté sur la forme des prestations de conseil aux PME/PMI}

La forme des prestations du conseil va naturellement dépendre de la façon dont il conçoit l'exercice de son métier. En l'absence fréquente d'un cahier des charges préalable précisant de façon détaillée les modalités pratiques de l'intervention (et même dans ce cas, il y a très fréquemment des «dérives » par rapport au canevas initial), la relation de conseil se déroule souvent dans un certain flou et une relative improvisation, en fonction de la demande du client, de la situation de l'entreprise, de l'humeur du dirigeant, etc.

Les prestations de conseil aux dirigeants de PME/PMI sont généralement caractérisées par leur faible formalisme bien que les rapports de mission écrits soient courants, car demandés par les organismes aidant financièrement la mission. Là, réside également une cause d'ambiguitté fréquente entre le conseil et le dirigeant de PMI. Le premier a l'impression d'avoir bien effectué sa mission lorsqu'il a remis un rapport détaillé et le chef d'entreprise retient surtout les apports concrets et techniques du conseil, ainsi que leur incidence directe et immédiatement tangible sur le fonctionnement et les résultats de l'entreprise. 
Les ambiguïtés évoquées ci-dessus conduisent à s'interroger, d'une part, sur la véritable valeur ajoutée d'une relation de conseil en management et, d'autre part, sur le décalage existant entre la valeur ajoutée émise consciemment et inconsciemment par le conseil et la valeur ajoutée réellement perçue consciemment et inconsciemment par le client dirigeant de PME/PMI. Une question centrale est donc d'apprécier quels sont les impacts des aspects objectifs, mais aussi et surtout des aspects subjectifs et psychologiques de cette relation entre le conseil et le dirigeant de PME/PMI.

Afin de clarifier la relation du conseil au dirigeant de PME/PMI et éviter certaines des ambiguïtés évoquées, nous proposons une approche «pédagogique» du conseil basée essentiellement sur les processus d'apprentissage personnels et collectifs que doit favoriser ce type de relation, tels que des changements de regard sur l'environnement de l'entreprise et de ses marchés, le recours à de nouveaux concepts pour la réflexion stratégique, l'adoption de nouveaux comportements managériaux vis-à-vis du personnel, de nouvelles visions de l'organisation de l'entreprise, la recherche de solutions différentes de celles qui ont prévalu jusqu'alors, par exemple, dans une optique systémique, etc.

Cette approche «pédagogique» du conseil aux dirigeants de PME/PMI permet d'éviter les obstacles fréquents liés à l'effort d'écoute d'un tiers censé apporter la «bonne parole». Il est en effet clair dans cette optique que le conseil en management a plus un rôle de catalyseur, de faire-faire que de faire à la place du dirigeant, ce qui est beaucoup plus valorisant au plan psychologique pour ce dernier.

Mais cette approche suppose, soulignons-le bien, un contexte favorable basé essentiellement sur une relation de confiance et d'estime entre le dirigeant de PME/PMI et le conseil sensiblement différente de la simple relation commerciale de client à prestataire qui régit souvent l'intervention d'un conseil dans l'esprit du dirigeant de PME/PMI.

C'est précisément tout l'art du professionnel compétent que d'établir au préalable ce contexte relationnel adéquat en expliquant sa démarche et en convainquant son interlocuteur de son intérêt et de ses avantages par rapport à des démarches de conseil plus «classiques».

\section{Activité de conseil aux PME/PMI et processus d'apprentissage du dirigeant}

\subsection{Relation de conseil et apprentissage}

Considérer la relation de conseil entre un intervenant extérieur et un dirigeant de PME/PMI en termes de processus d'apprentissage permet d'ouvrir d'intéressantes perspectives pour mieux comprendre la nature profonde de la relation 
de conseil, pour dissiper de nombreuses ambiguïtés (parmi celles évoquées précédemment) et enfin pour contribuer à renouveler les pratiques du métier de conseil.

Le concept «d'apprentissage » est en général mal connu dans le domaine du management. Il a été trop souvent en France confondu avec la formation des apprentis et en a subi des connotations péjoratives injustifiées. Or, l'apprentissage a un sens beaucoup plus large et riche de significations qu'une simple situation didactique. Les récits d'apprentissages montrent bien qu'ils interviennent à travers la vie, les expériences sociales et professionnelles, les rencontres individuelles, les voyages, les groupes, les échecs et les succès, etc. ${ }^{9}$.

Le célèbre psycho-pédagogue américain Rogers (1972) a souligné l'intérêt de l'apprentissage par rapport à l'enseignement. Selon lui, l'important est que l'apprentissage parte de l'individu qui apprend, l'engage tout entier et le transforme dans ses attitudes, ses comportements et sa personnalité. Il procède donc d'une autotransformation et d'une autoconstruction de l'apprenant. Il propose une action thérapeutique centrée sur le client, une pédagogie centrée sur l'élève où le maître joue seulement un rôle de catalyseur, de «facilitateur» dans l'apprentissage de l'élève. Il propose une inversion fondamentale qui met l'élève au centre de la situation pédagogique et à la source du processus d'apprentissage. Le maître est au service du développement de l'élève sans négliger pour autant les connaissances, les émotions et les apprentissages du maître.

Il paraît très pertinent de reprendre ce concept d'apprentissage développé par Rogers dans un contexte psycho-pédagogique scolaire pour le transposer à une relation de conseil entre un intervenant extérieur et un dirigeant de PME/PMI. Dans cette perspective, le conseil n'est plus l'expert ou le détenteur du savoir venant le transmettre sous une forme ou une autre à son client moyennant rémunération. Il devient avant tout le catalyseur, le facilitateur d'apprentissage de celui-ci. Cette optique du conseil induit donc des finalités et des pratiques professionnelles très différentes axées sur la gestion des processus d'apprentissage du client et plus seulement sur l'apport d'un contenu d'expertise technique. C'est le «process consulting» des Anglo-Saxons pratiqué par des auteurs comme Schein ${ }^{10}$ par opposition aux classiques «resources consulting » des grands cabinets-conseils en management américains.

9. Voir à ce sujet Clouzot et Bloch (1981) et Dominice (1990).

10. Les aspects conceptuels du «process consulting» et de l'approche «clinique» du conseil d'entreprise ont été exposés par Schein (1969 a et b). On peut remarquer également que pour les promoteurs de l'approche dite du «Développement organisationnel », la nature même du conseil serait la relation entre le consultant et le client. Voir à ce sujet notamment Gallessich (1983) et Greiner et Metzger (1983). 
Cette autre conception du conseil d'entreprise, axée sur les processus, a le mérite de viser à une transformation en profondeur des perceptions, des modes de pensée et des comportements du responsable d'entreprise par une gestion de ses processus d'appropriation et d'apprentissage, afin qu'il découvre lui-même les meilleures solutions à ses problèmes.

Elle s'oppose à la délivrance d'un message d'expert extérieur qui a l'inconvénient de rester souvent sans grand impact sur le dirigeant de PME/PMI, car il ne changera pas en profondeur sa perception des choses et ses façons de raisonner.

Dans cette approche en termes d'apprentissage, il convient de souligner le rôle de «l'autre». Comme le font remarquer Clouzot et Bloch (1983), si l'apprentissage est personnel et si on ne peut apprendre à la place de quelqu'un d'autre, nous apprenons souvent avec l'autre, grâce à lui ou à cause de lui. «L'autre» est au cœur de nos apprentissages à travers plusieurs rôles possibles parfois simultanés ou consécutifs. Ces auteurs distinguent notamment parmi ces rôles : le médiateur, le confident, le compagnon de route, le questionneuraccoucheur, le transmetteur de connaissance, l'évaluateur, l'éveilleur et le guide, repris par Aubrey (1990).

Ce rôle de «l'autre» dans le processus d'apprentissage peut être parfaitement transposé au rôle du conseil d'entreprise dans sa relation avec son client. On retrouve parmi les rôles cités plusieurs des fonctions exercées par un intervenant extérieur vis-à-vis de son client à des degrés divers (et souvent de façon plus ou moins consciente). On observera que ces rôles reflètent d'ailleurs en eux-mêmes plusieurs styles de conseil d'entreprise, sans qu'ils procèdent pour cela d'une volonté de l'intervenant de gérer des processus d'apprentissage individuels et collectifs.

Une autre théorie intéressante sur le concept d'apprentissage est celle de l'approche systémique ou école dite de «Palo Alto» (Marc et Picard, 1984). C'est en particulier Bateson qui a montré que tout comportement résulte d'un processus d'apprentissage et que les apprentissages résultent largement du contexte interactionnel dans lesquels ils s'opèrent. Sans entrer ici dans le détail de la théorie de l'apprentissage de Bateson, retenons que des changements profonds de comportement à travers des processus d'apprentissage ne peuvent résulter que de modifications des contextes d'apprentissage et de changement du système dont ils sont issus.

En pratique, cet auteur indique que cela ne peut se faire que par une modification du contexte conceptuel et émotionnel d'une situation et du point de vue selon lequel elle est vécue, en la plaçant dans un autre cadre qui soit neutre ou corresponde mieux aux faits. Le sens de cette situation concrète change complètement. Il s'agit d'adapter la personne au réel, conçu comme 
perception subjective qui tient compte de la valeur accordée aux choses et aux faits. La situation, dans un nouveau contexte, conserve en remaniant les anciens éléments dans un rapport complètement différent sur le plan conceptuel.

Cette approche puissante des phénomènes d'apprentissage peut être aussi très directement transposée à la relation de conseil entre un intervenant extérieur et un dirigeant de PME/PMI. Elle ouvre la voie à des principes et des pratiques d'intervention très différentes des pratiques courantes axées essentiellement sur la gestion des contextes d'apprentissage, ainsi que sur la modification de la perception et du sens de l'univers dans lequel on se situe.

Dans cette perspective systémique, le conseil a pour rôle essentiel de faire voir le monde et les problèmes perçus avec «d'autres lunettes » en transformant le contexte conceptuel et émotionnel dans lequel se situe son client.

Une illustration intéressante de cette pensée systémique est présentée par Senge (1991) à travers le concept grec de «metanoia» qui signifie changement d'état d'esprit. Selon lui, «le vrai apprentissage nous permet de nous recréer, de devenir capable de faire ce qui nous était avant impossible, de modifier notre manière de voir le monde et nos relations avec ce dernier». Il précise que le raisonnement systémique est fondé essentiellement sur un changement de mentalité, de vision et de perception des organisations. De plus, il montre bien dans cette approche systémique que nos modes de pensée et nos comportements sont souvent prisonniers de structures mentales et humaines que nous ne saisissons pas, mais qui déterminent nos perceptions, nos décisions, nos actions, nos objectifs, nos normes, etc. De plus, nous comprenons souvent mal les effets de nos propres comportements qui s'avèrent être la source essentielle de nos problèmes.

Ce raisonnement systémique apparaît comme une philosophie très intéressante non seulement au plan d'une théorie de l'action pour le métier de conseil, mais aussi pour enclencher des processus d'apprentissage profonds débouchant sur d'autres visions du monde et des problèmes des entreprises plus pertinentes et donc susceptibles d'ouvrir la voie à des solutions pratiques innovantes et plus efficientes.

Si l'on a mis l'accent dans cet article sur les processus d'apprentissage individuels naissant de la relation de conseil, il convient de souligner qu'en général ceux-ci sont démultipliés par des processus d'apprentissage collectifs au sein de l'entreprise. Ceux-ci interviendront surtout dans la mesure où il y a volonté du dirigeant de diffuser ses apprentissages à travers l'entreprise.

Ce phénomène sera amplifié et son efficacité accrue s'il existe également une volonté, des compétences pédagogiques et des procédures de formation appropriées pour sensibiliser, expliquer, convaincre et faire passer le message auprès de l'ensemble des hommes de l'entreprise (Garratt, 1989 et 1990). 


\subsection{Le rôle pédagogique du conseil dans sa prestation auprès de la PME/PMI}

On constate que le métier de conseil est en pratique très souvent associé à celui de formateur dans sa présentation, mais aussi dans sa pratique. Bon nombre de cabinets-conseils font également de la formation et de nombreux organismes de formation font aussi du conseil auprès des entreprises. En réalité, il paraît bien difficile et inutile de vouloir tracer des frontières entre les prestations de conseil et les prestations de formation, surtout dans le contexte des PME/PMI. La pratique de ces métiers montre bien que ces distinctions ont surtout des raisons administratives ou commerciales et, qu'au fond, il existe une continuité et une interrelation profondes entre ces deux types de prestations.

Pour de nombreux auteurs, comme par exemple Romano (1991), le conseil est d'ailleurs indissociable de la formation. Certes, la distinction sera plus ou moins nette en fonction des différentes conceptions et modalités pratiques d'exercice du métier de conseil auprès des PME/PMI des intervenants. Le rôle pédagogique du conseil est en fait très variable et lié à sa personnalité, à son mode pratique d'intervention, au climat relationnel établi avec le dirigeant d'entreprise et au degré de confiance existant entre les interlocuteurs. Il peut être quasi nul dans certains cas à relativement important dans d'autres, et ce, quel que soit son profil. L'absence d'expertise psycho-pédagogique de la vaste majorité des conseils d'entreprise (ainsi que de très nombreux formateurs) intervenant auprès des PME/PMI explique aussi la faiblesse de cette dimension pédagogique de la relation avec le dirigeant d'entreprise et ses conséquences.

Certes, il convient d'observer que cette fonction pédagogique du conseil d'entreprise est particulièrement délicate à exercer surtout avec des dirigeants de PME/PMI qui ont appris leur métier la plupart du temps sur le terrain par la confrontation avec les problèmes quotidiens de leur entreprise. Le caractère autodidactique de leur formation professionnelle constitue souvent un obstacle aux nécessaires apprentissages à faire pour remettre en cause les attitudes et comportements traditionnels. Ce phénomène doit être intégré dans la réflexion sur des approches pédagogiques du conseil et de la formation appropriées aux caractéristiques spécifiques de cette population de dirigeants.

Comme le montrent les cycles de formation et de perfectionnement au management destinés à ces dirigeants de PME/PMI, il existe de sérieuses difficultés psychologiques lorsqu'on veut faire passer un message de façon trop didactique. On constate un rejet du système éducatif de type scolaire traditionnel et de la relation maître-élève, des approches trop théoriques ou conceptuelles des problèmes de l'entreprise, et des intervenants extérieurs jugés souvent trop éloignés de la réalité des problèmes de terrain auxquels ces dirigeants de PME/PMI sont quotidiennement confrontés. 
Cependant, il existe implicitement au moins une demande de nature pédagogique dans les sollicitations de conseil de bon nombre de dirigeants de PME/PMI. Mais celle-ci est mal perçue et la plupart du temps mal traitée par des intervenants non préparés à cette dimension de leur métier de conseil.

Il semble donc nécessaire à l'avenir d'imaginer de nouvelles approches du conseil aux dirigeants de PME/PMI plus en rapport avec leur psychologie, leurs perceptions, leurs expériences et surtout leurs processus et styles d'apprentissage propres, par exemple, selon les concepts développés par Honey et Munford (1984) et Kolb (1986).

\subsection{Quelle est la valeur ajoutée réelle d'une relation de conseil en management?}

En réalité, le véritable rôle du conseil d'entreprise n'est-il pas d'abord de créer les conditions nécessaires à l'émergence des bonnes solutions à travers une dynamique d'appropriation du diagnostic et d'une réflexion sur les changements à introduire par les acteurs? (et d'abord l'acteur principal qu'est le dirigeant ?) N'est-il pas ensuite, si possible, d'accompagner ce processus de changement dans sa concrétisation sur le terrain?

De façon schématique, le rôle du conseil, dans une optique d'apprentissage, doit être essentiellement d'impulser les processus d'apprentissage individuels (et d'abord du dirigeant) et collectifs (l'ensemble du personnel de l'entreprise) nécessaires aux transformations souhaitables du management de l'entreprise, et de veiller à leur opérationnalisation au sein de l'organisation et des modes de fonctionnement de l'entreprise.

Cette approche «pédagogique» de la relation de conseil entre l'intervenant et son client PME/PMI est indissociable d'une réflexion sur les spécificités des processus d'apprentissage du dirigeant d'entreprise qui résultent de cette relation. Dans cette perspective, le rôle du consultant consiste à susciter, développer et guider les processus d'apprentissage individuels de son client (en l'aidant notamment à définir et à valider les nouveaux principes d'action qu'il se sera fixés) et à s'assurer de leur démultiplication collective au niveau de l'ensemble de l'entreprise.

Cette autre philosophie du conseil en management auprès des dirigeants de PME/PMI a pour principal mérite de mieux appréhender les dimensions psychologiques et de communication des relations de conseil avec toutes les difficultés qui en résultent et qui sont, comme nous l'avons souligné, la source de la plupart des multiples ambiguïtés qui caractérisent l'exercice de ce métier.

Prenons, par exemple, le cas évoqué des ambiguïtés liées aux attentes du dirigeant de PME/PMI vis-à-vis de l'intervention d'un conseil appelé pour des 
prestations de conseil en stratégie. Dans la perspective classique du conseilexpert, le client attendra du conseil, outre un diagnostic pertinent, la formulation argumentée d'un plan stratégique pour son entreprise et, le cas échéant, un certain suivi de sa mise en place dans l'entreprise. Mais il est clair, dans cette optique, que le degré d'implication du client a des chances d'être très variable ainsi d'ailleurs que l'application réelle des préconisations du conseil. De plus, le client pourra facilement critiquer les recommandations faites ou trouver diverses raisons pour ne pas les suivre...

Dans l'optique du conseil-pédagogue proposée ici, le conseil aura une approche très différente du problème posé. Son objectif principal sera de faire en sorte que ce soit le dirigeant d'entreprise lui-même qui effectue le diagnostic stratégique de l'entreprise et qui définisse son plan stratégique. Son rôle consistera alors à lui apporter les supports conceptuels et méthodologiques nécessaires et à l'aider et le guider dans sa réflexion pour qu'il puisse atteindre l'objectif fixé. On constate dans cette démarche "pédagogique» que le conseil doit certes avoir une expertise technique dans son domaine d'intervention, mais surtout aussi une expertise pédagogique pour guider le processus d'apprentissage de son client. L'ambiguîté vis-à-vis des prestations du conseil disparaît, car il est clair dès le départ que ce dernier n'intervient pas pour apporter la solution, puisque sa mission consiste à aider son client à la trouver lui-même et à la mettre en pratique. Ce qui est également beaucoup plus valorisant et facile à admettre sur le plan psychologique pour le client. De plus, cette approche a le mérite de susciter l'appropriation des changements décidés par le dirigeant lui-même et, par conséquent, a beaucoup plus de chances de se traduire dans les faits qu'un simple apport d'expertise extérieure, aussi pertinent soit-il. Enfin, l'apprentissage personnel qu'il aura permis donnera la possibilité au dirigeant de mieux faire face à ses futurs problèmes de stratégie d'entreprise et réduira sa dépendance vis-à-vis de sources d'expertise extérieures, en général, coûteuses.

On voit que cette démarche du conseil en management met davantage l'accent sur la gestion des processus d'apprentissage de son client et moins sur l'apport externe d'expertise technique et de contenus en termes d'apport de solutions toutes faites. Toutefois, précisons bien qu'il ne s'agit pas ici de sous-estimer l'utilité et l'intérêt de prestations de conseil en termes d'apport d'expertise pour résoudre des problèmes techniques ponctuels de gestion, au contraire. Mais il convient de bien distinguer, d'une part, les prestations de conseil en gestion relevant des différentes techniques ou disciplines de gestion et, d'autre part, celles du conseil en management procédant essentiellement des principes de direction générale et du style de management propre au dirigeant de l'entreprise (et qui ont souvent un poids particulier dans le contexte des PME/PMI). 
Cette autre approche du métier de conseil en management permet par ailleurs d'ouvrir des horizons nouveaux pour renouveler et professionnaliser davantage ce métier à l'avenir comme nous le préciserons ultérieurement.

\subsection{Pour une relation de conseil aux dirigeants de PME/PMI fondée sur leurs processus d'apprentissage}

Nous avons tenté de montrer que la relation de conseil qui s'établit entre un intervenant extérieur et un dirigeant de PME/PMI devrait comprendre essentiellement une dimension d'apprentissage. Aussi, afin de rendre cette relation plus performante, paraît-il nécessaire d'essayer de comprendre la réalité et la nature des processus d'apprentissage managériaux des dirigeants de PME/PMI.

Remarquons d'abord que la connaissance des processus d'apprentissage des gestionnaires et des dirigeants d'entreprises constitue aujourd'hui un domaine d'intérêt et de recherche majeur pour la conception d'outils pédagogiques dans le domaine du management ${ }^{11}$. En effet, l'enjeu essentiel des actions de conseil et de formation est bien en général de susciter des changements de mentalité, d'attitudes et de comportements à travers de nouveaux apprentissages.

En effet, seule une appréhension correcte des processus d'apprentissage des dirigeants de PME/PMI permettra d'élaborer des «stratégies pédagogiques » de conseil et de formation efficaces. Des contributions récentes ont essayé de préciser la nature des processus d'apprentissage des dirigeants.

Mintzberg (1973 et 1989) a étudié les modes d'apprentissage des dirigeants et a souligné le rôle de l'intuition et de l'expérience dans leur formation. Isenberg (1985) a mis en évidence l'importance des processus de réflexion «irrationnels» et de l'utilisation de l'intuition dans les modes de pensée des dirigeants et l'existence d'une dialectique complexe entre la réflexion et l'action. Il montre les savoirs pratiques mobilisés par les dirigeants et leur fondement dans les actions et expériences quotidiennes, ainsi que la difficulté de rendre compte des processus de cognition des dirigeants à l'aide de concepts issus de la séparation entre action et connaissance qui prévaut dans les doctrines et les formations au management.

Bouchikhi (1991) décrit les principales caractéristiques des processus d'apprentissage du métier de dirigeant. Il observe que les processus d'apprentissage liés au métier de dirigeant émergent progressivement de la théorie de

11. Il convient de citer notamment les initiatives du groupe ESC Lyon en liaison avec l'Université de Lancaster en matière de pédagogie du management avec l' « European Management Teacher Program » et le forum sur « les innovations pédagogiques pour la formation au management » des 9 et 10 avril 1992. 
l'action du dirigeant, qu'ils ont un caractère incrémental, qu'ils sont influencés par les schémas mentaux issus des expériences passées et que chaque dirigeant construit progressivement sa propre théorie managériale dans l'action de façon contingente, en fonction des situations auxquelles il a été et est confronté. Il remarque aussi que certains individus cessent d'apprendre à un moment donné, ayant une confiance trop grande dans leur jugement et leur façon d'agir, et qu'il existe des processus d'apprentissage collectifs avec le groupe des proches collaborateurs.

Même si cette analyse des processus d'apprentissage des dirigeants est issue de l'étude biographique de dirigeants célèbres de grandes organisations, il semble à l'auteur, au vu de son expérience, qu'elle soit également pertinente pour les dirigeants de PME/PMI.

Leurs processus d'apprentissage se caractérisent notamment par le rôle de l'expérience concrète, l'observation perspicace de l'environnement et du marché, l'action par essais et erreurs, la réactivité, l'apprentissage en petit groupe, la confrontation permanente dans l'action avec des situations nouvelles, les théories personnelles de l'action managériale, etc.

La connaissance des caractéristiques de ces processus d'apprentissage des dirigeants (et en particulier des dirigeants de PME/PMI) a une importance primordiale pour réfléchir à la forme et au contenu des relations de conseil à leur égard. L'intervenant qui veut aider le dirigeant d'entreprise par une action de conseil doit intégrer dans sa «stratégie pédagogique» les spécificités de leurs processus d'apprentissage de nature individuelle et collective. Ceux-ci constituent des leviers pour agir et influencer leurs attitudes, leurs comportements, leurs raisonnements et, en un mot, leur «théorie de l'action» managériale.

Cette approche permet de s'interroger sur les pratiques courantes du conseil et leur degré d'efficacité. On a déjà souligné la distinction entre, d'une part, les formes de conseil de type «expert» apporteur d'une solution technique extérieure (ou «conseil-contenu») et, d'autre part, les formes de conseil de type «mentor» se caractérisant par une aide à la réflexion du dirigeant afin qu'il trouve lui-même les solutions à ses problèmes (ou «conseil-processus») ${ }^{12}$.

Il est clair qu'une conception du conseil en termes d'apprentissage individuel du client privilégiera la seconde approche beaucoup plus cohérente avec les caractéristiques des processus d'apprentissage des dirigeants évoquées et

12. Remarquons que cette caractéristique des processus d'apprentissage des dirigeants d'entreprise s'inscrit dans le cadre d'une épistémologie de type «constructiviste» dont l'origine philosophique remonte à G.B. Vico et qui se retrouve notamment dans les travaux de G. Bachelard et J. Piaget. 
les mécanismes d'appropriation nécessaires à des effets réels sur leurs attitudes et comportements. Selon cette optique du «conseil-processus», la relation de conseil qui s'établit entre l'intervenant extérieur et le dirigeant de PME/PMI peut s'analyser en termes de sensibilisation, de catalyse, de guide et d'accompagnement des apprentissages du client.

Remarquons que cette «théorie pédagogique» du conseil aux dirigeants de PME/PMI s'applique particulièrement bien aux domaines du conseil en management et stratégies d'entreprise, domaines complexes et multidimensionnels qui nécessitent une réflexion globale et à moyen terme sur l'entreprise et son environnement, ainsi que la responsabilité essentielle du dirigeant. Elle conduit à une révision profonde et générale de l'ensemble des principes d'action et des pratiques professionnelles courantes du métier de conseil en management, en particulier, vis-à-vis des PME/PMI.

\section{Vers une autre approche du métier de conseil en management aux PME/PMI}

\subsection{Pour une philosophie "andragogique» du métier de conseil en management}

Si nous avons souligné la dimension pédagogique implicite et fondamentale de toute relation de conseil, nous avons également mis en évidence les sérieux obstacles psychologiques existant au niveau des dirigeants de PME/PMI face aux approches didactiques classiques, que ce soit en matière de conseil ou de formation, et par conséquent, toutes les ambiguittés et difficultés qui en résultaient.

Aussi, paraît-il particulièrement intéressant de s'inspirer des apports du modèle «andragogique» de la formation (Schein, 1987) pour développer la dimension "pédagogique» du métier de conseil (mais aussi de formateur) aux dirigeants de PME/PMI. Ce modèle «andragogique» a été très clairement présenté par Knowles (1990) ${ }^{13}$ qui l'oppose au modèle «pédagogique » classique d'inspiration behavioriste dont une des faiblesses est de ne pas prendre en compte correctement les spécificités des processus d'apprentissage des adultes. Dans ce «modèle andragogique» de l'apprentissage, Knowles pose les hypothèses suivantes sur le comportement «d'apprenant» des adultes (définis

13. L'andragogie est définie ici comme l'art d'enseigner à des adultes par référence à la «pédagogie » qui est l'art d'enseigner à des enfants ou adolescents. 
psychologiquement comme des personnes ayant pris conscience qu'ils sont responsables de leur vie et de la nécessité de s'autogérer) :

- rôle essentiel de l'expérience de l'apprenant;

- aspect utilitaire de l'apprentissage (mieux affronter des situations réelles);

- orientation de l'apprentissage autour de la vie, d'un problème ou d'une tâche donnée;

- assimilation des connaissances et des savoir-faire plus facile lorsque présentés dans le contexte de leur mise en application à des situations réelles;

- motivations d'apprentissage liées à la connaissance des objectifs poursuivis et à des facteurs intrinsèques propres à l'apprenant.

On constate que ce modèle "andragogique» peut très bien s'appliquer aux relations de conseil entre l'intervenant extérieur et le dirigeant d'entreprise dans la perspective proposée ici de développement de l'apprentissage de ce dernier.

Dans cette perspective andragogique, le rôle du conseil d'entreprise est essentiellement d'être le catalyseur et le guide des processus d'apprentissage de nature individuelle (le dirigeant de PME, ses collaborateurs) et aussi collective (l'ensemble du personnel de l'entreprise). Il facilitera le processus d'apprentissage de son client en l'aidant dans sa réflexion (questionnement, apports méthodologiques, apports de nouveaux concepts, d'expériences d'entreprises, etc.), en jouant un rôle de «mentor», voire de miroir, en l'accompagnant dans son processus de remise en question de ses représentations et de transformation de ses perceptions et structures mentales.

En un mot et de façon schématique, sa prestation consistera à faire en sorte que son client se pose les bonnes questions, au bon moment, et trouve lui-même les solutions appropriées au lieu de chercher à apporter sa solution d'expert.

On remarquera que l'application de cette théorie «andragogique» du conseil d'entreprise débouche sur une théorie de l'apprentissage managérial du dirigeant. Elle apparaît comme tout particulièrement pertinente pour des réflexions globales sur l'entreprise comme celles relatives au management stratégique, à l'organisation et au management des ressources humaines qui doivent être celles d'un dirigeant d'une PME/PMI. En outre, elle a le mérite d'ouvrir de nouvelles perspectives méthodologiques pour le perfectionnement au management des responsables d'entreprises (Knowles, 1990). 


\subsection{Vers d'autres principes d'action pour l'exercice du métier de conseil en management auprès des PME/PMI}

La philosophie du conseil d'entreprise précédemment esquissée conduit à une autre conception de ce métier et à d'autres principes d'action que ceux qui dominent aujourd'hui cette profession. Sans pouvoir ici évoquer de façon détaillée les modalités pratiques et méthodologiques de ce métier, ni analyser les variantes de style d'intervention liées à la personnalité de chaque conseil, nous suggérons ci-après quelques-uns des principes $d$ 'action et domaines de compétences qui devraient caractériser l'exercice du métier de conseil en management selon cette philosophie «pédagogique»:

- Il est compétent pour percevoir les spécificités de la personnalité de son client, prendre en compte ses expériences et apprentissages passés, appréhender ses motivations, ses capacités et ses modes principaux d'apprentissage (Belet, 1991).

- Il a un rôle de guide et de catalyseur du processus d'apprentissage de son client. Il n'a pas pour rôle principal d'apporter des solutions en termes de contenu, mais il l'aide à faire émerger les bonnes solutions en le guidant dans son processus de réflexion.

- Il appuie sa démarche "pédagogique» sur les problèmes concrets de l'entreprise, l'expérience de son dirigeant, la nécessité de l'action. Il ne dissocie pas les aspects théoriques et pratiques intimement mêlés dans l'appréhension de situations réelles d'entreprise.

- Il favorise une réflexion inductive et conceptuelle de son client à partir des réalités du terrain et une approche globale des problèmes au lieu de raisonner par discipline de gestion et de façon déductive à partir d'une quelconque théorie de management.

- Il apporte aussi de nouvelles visions des choses, de nouveaux concepts, de nouveaux regards sur le fonctionnement du monde non pas comme solutions, mais comme autant de stimulants pour la réflexion de son client.

Il l'aide par ses points de vue à se poser les bonnes questions, à découvrir la nature des systèmes de pensée où il évolue, à en envisager d'autres lui permettant de découvrir les vraies causes des problèmes et les dimensions de leur complexité ${ }^{14}$.

14. Il semble intéressant d'avoir aussi une lecture «constructiviste » de la démarche d'apprentissage du dirigeant de PME/PMI, car l'action précède souvent la réflexion et sa théorie de l'action résulte essentiellement de ses expériences de terrain (voir Bachelard et Piaget). 
Il favorise ainsi l'émergence de vues globales et à long terme de son client, de raisonnements en termes d'interrelations plutôt que de façon analytique à partir d'éléments disjoints et parcellaires empêchant d'appréhender les problèmes dans la réalité de leur complexité et de leur dynamique.

Il joue un rôle actif pour faire évoluer les représentations et modèles mentaux de son client en favorisant des processus de transformation psychologiques basés sur l'appropriation (Morin, 1990) au lieu d'apporter de l'extérieur de nouveaux schémas et de chercher à les plaquer sur l'entreprise où il intervient. Dans cette perspective, le conseil devient un «architecte de la théorie d'action » du dirigeant et un pédagogue agissant au niveau des structures mentales de son client. Il l'aide par son intervention à dissiper les éléments de blocage de ses processus d'apprentissage, à «reconstruire» de nouveaux modèles mentaux et de nouveaux modes de raisonnements qui lui permettront de mieux résoudre les problèmes concrets et évolutifs auxquels il est confronté quotidiennement dans son entreprise.

\subsection{L'exigence de compétences professionnelles spécifiques pour l'exercice de ce nouveau métier de conseil en management}

Ces nouveaux principes d'action et les pratiques professionnelles auxquelles ils conduisent impliquent la maîtrise de solides compétences techniques en gestion et management et surtout la possession de compétences psychologiques, pédagogiques et de communication.

Or, on observe que la grande majorité des praticiens, notamment en milieu de PME/PMI, ne possède pas de telles compétences de par leurs origines et leurs expériences professionnelles. De plus, ils ont en général une conception de leur métier et de la nature de leurs prestations essentiellement orientée vers les contenus, vers des préconisations en termes de «il faut faire ceci ou cela et ne pas faire ceci ou cela». Ils ont une approche «thérapeutique» du métier de conseil, alors que, selon la perspective proposée, elle devrait être surtout de nature «psycho-pédagogique».

Selon cette nouvelle optique du métier de conseil aux dirigeants de PME/PMI, on perçoit qu'à l'avenir les compétences nécessaires à l'exercice performant du métier de conseil en management devraient être sensiblement différentes de celles qui prévalent actuellement. Elles devraient comprendre:

- d'une part, de solides connaissances techniques de "généraliste en gestion», afin d'avoir une vue globale et pertinente des problèmes de l'entreprise et des interrelations existantes entre leurs différentes 
facettes (commerciale, production, R-D, logistique, achats, finances, ressources humaines, etc.),

- d'autre part, et surtout, des compétences psychologiques, pédagogiques et de communication afin d'appréhender les caractéristiques psychologiques et comportementales du dirigeant, ses représentations et modèles mentaux, ses processus d'apprentissage, et d'être capable d'agir sur ces processus pour l'aider à trouver des solutions personnelles aux vrais problèmes qu'il rencontre dans son entreprise.

L'expertise technique caractérise en principe les praticiens du conseil (Godet, 1991). Elle a pour origine une formation initiale supérieure à la gestion (grandes écoles, universités) ou de cycles de formation continue ( tels que IAE, ICG, CESI, etc. dans le contexte français) et/ou une expérience professionnelle acquise en entreprise.

En revanche, la seconde catégorie de compétences d'ordre psycho-pédagogique et de communication n'a, sauf dans certains cas particuliers, pas fait l'objet d'un apprentissage spécifique de la part des conseils en management, notamment ceux qui interviennent couramment auprès des PME/PMI. Certes, il se trouve que certains consultants ont intuitivement certaines dispositions dans ces domaines liées à leur personnalité et à leur style propre d'intervention. Mais il ne s'agit pas de réelles compétences professionnelles ayant fait l'objet d'un apprentissage théorique et pratique spécifique.

Ce phénomène s'explique d'abord au niveau des formations initiales par les particularités du système d'enseignement supérieur français avec les cloisonnements existants entre, d'une part, les sciences de gestion, les sciences du comportement et les sciences de la communication. Il s'explique aussi par les schémas mentaux dominants de type «utilitariste» caractérisant l'univers des entreprises de leurs dirigeants et, par conséquent, des prestataires de services évoluant dans ce domaine. Il s'explique enfin par le caractère encore juvénile du métier de conseil d'entreprise et la relative faiblesse des réflexions théoriques et philosophiques sur ses différentes dimensions et ses finalités.

Aussi, paraît-il opportun, selon cette nouvelle vision du métier de conseil aux dirigeants de PME/PMI, de concevoir de nouvelles formations préparant de façon plus judicieuse à ce métier et permettant de déboucher sur les «qualifications professionnelles» de haut niveau qu'il requiert, et requerra sans doute davantage encore à l'avenir ${ }^{15}$.

15. Il se développe des labels de qualification reconnus au plan national dans cette profession du conseil non réglementée tel que celui de l'OPQCM (Office professionnel de qualification des conseils en management) qui bénéficie du soutien des pouvoirs publics. 
De telles formations contribueraient à développer un nouveau type de professionnalisme pour le métier de conseil (notamment auprès des PME/PMI) et à améliorer l'image de ce métier au plan de sa crédibilité et de sa respectabilité, tant aux yeux des dirigeants d'entreprises qu'à ceux des différents partenaires de l'entreprise et des pouvoirs publics.

\section{Bibliographie}

ATAMER et al. (1989), «L'État, les PME et les consultants. Le marché de la confiance», Revue Gérer et Comprendre. Annales des Mines, juin, p. 28-37.

Aubrey, B. (1990, Savoir Faire Savoir, Paris, InterÉditions.

BELET, D. (1991), «Changer les comportements managériaux par la formation: pour une andragogie du management », CREGE, Université de Bordeaux 1, Travaux de recherches $n^{\circ} 1-9103$.

Bernard-Julhiet Conseils (1991), Les PMI et l'aide au conseil, Paris.

BLANC, G. (1989), «Le profil du consultant», Revue française de Gestion, $n^{\circ} 76$, novembre-décembre, p. 82-86.

BoUCHIKHI, H. (1991), «Apprendre à diriger en dirigeant », Revue Gestion, novembre, p. 56 à 63.

Bounfour, A. (1989), Les conseils en management, Paris, Rivages-Les Échos.

Bounfour, A. (1989), «Vers l'industrialisation du conseil», Revue française de Gestion, ${ }^{\circ} 76$, novembre-décembre, p. 23-32

Bounfour, A. (1992), Chers consultants, Paris, Dunod.

Clouzot, O. et A. Bloch (1981), Apprendre autrement, Paris, Éditions d'Organisation.

DETRIE, J.P. (1989), « Paysages du conseil », Revue française de Gestion, n 76, novembre-décembre, p. 6-14.

DOMINICE, P. (1990), L'histoire de vie commune processus de formation, Paris, Éditions l'Harmattan.

ENGEL, F. et D. FIXARI (1987), «Les consultants et les PME. L'impact de l'aide au conseil », Ministère de l'Industrie, Centre de gestion scientifique de l'École des Mines, Paris, novembre.

FinANCIAL TIMES SURVEY (1988), «Management Consultancy », 5 octobre.

GallessiCH, J. (1983), The Profession and Practice of Consultation, New York, JosseyBass Publishers. 
GARRATT, B. (1989), The Learning Organization, Fontana, Harper Collins.

GARRATT, B. (1990), Learning to Lead, Fontana, Harper Collins.

Gastou, G. et M. Thevenet (1989), «L'abeille et la muse » Revue française de Gestion, $\mathrm{n}^{\circ} 76$, novembre-décembre, p. 67-72

GODET, M. (1991), De l'anticipation à l'action, Manuel de prospective et de stratégie, Paris, Dunod.

GREINER, L. et D. NEES (1989), « Conseils en management : tous les mêmes ?», Revue française de Gestion, ${ }^{\circ} 76$, novembre-décembre, p. 55-66.

Greiner, L. et R. METzGer 1983), Consulting to Management, Englewood Cliffs, N.J., Prentice-Hall.

Honey, P. et A. MuMford (1986), The Manual of Learning Styles, 2e éd., Berks, Honey-Maidenhead.

ISENBERG, D.J. (1985), «Comment réfléchissent les dirigeants ?», Harvard-L'Expansion, été, p. 18-29.

KAHN, A. (1989), «Le conseil, florissant mais dispersé», Le Monde, 25 avril 1990 dossier spécial «Le Monde des consultants».

KNOWLES, M.S. (1990), L'apprenant adulte: vers un nouvel art de la formation, Paris, Éditions d'Organisation.

KolB, D.A. (1984), Experiential Learning, Englewood Cliffs, N.J., Prentice-Hall.

KUBR, M. (1981), Le conseil en management-guide pour la profession, $2^{\mathrm{e}}$ éd., Genève, BIT.

MARC, E. et D. PICARD (1984), L'École de Palo Alto, Paris, Éditions Retz.

MINTZBERG, H. (1973), The Nature of Managerial Work, New York, Harper and Row.

MinTzBerg, H. (1989), Mintzberg on Management, New York, The Free Press.

MORIN, E. (1990), Introduction à la pensée complexe, Paris, ESF Editeur.

NOYE, D. et E. VERNE (1991), Choisir un consultant, AFNOR Gestion.

ROGERS, C. (1972), Liberté pour apprendre, Paris, Dunod.

ROMANO, J. (1991), «Démarche qualité et innovation de formation, fonctions et usages du conseil aux PME-GREE », Université Nancy 2, octobre, p. 45.

SAUviat, C. (1989), «Les mutations du marché de l'expertise et du conseil en France », Revue de l'IRES, $\mathrm{n}^{\circ}$ 5, hiver, p. 3-32.

SAVALl, H. (1989), «Professeur-consultant ; le bilan d'une expérience», Revue française de Gestion, $n^{\circ} 76$, novembre-décembre, p. 93-103. 
SCHEIN, E.H. (1969), Process Consultation. Its Role in Organization Development, Reading (Mass.), Addison Wesley.

SCHEIN, E.H. (1987), Process Consultation, Volume 2, Lessons for Managers and Consultants, Reading (Mass.), Addison Wesley.

SENGE, P. (1991), La $5^{e}$ discipline : l'art et la manière des organisations qui apprennent, New York, First.

Stevenson, H. et W. SAhlman (1988), « How small companies should handle advisers », Harvard Business Review, mars-avril. 